\title{
Evaluation of Changes in Weed Flora in Response to Agricultural Practices in the Arable Lands of El-Menoufia Governorate, Nile Delta, Egypt
}

\author{
EISaied, A.* and Bedair, R.
}

Botany and Microbiology Dept., Faculty of Sciecnce (Boys), Al-Azhar University

*Corresponding author. Baraa_elsaied@yahoo.com. Tel.: +20 1012890605.

\begin{abstract}
In Egypt and during the last 50 years, several changes in agricultural practices have affected different components of agro-ecosystems including weed flora. The aim of the present study was to evaluate changes in the weed flora of El-Menoufia Governorate, South of the Nile Delta of Egypt during the period from 1984 to 2018, in response to different agricultural practices. A total of 240 stands were investigated in both spring and summer seasons in order to document the present status of the weed flora. The obtained results were compared to results obtained by other authors in 1984. Results showed major shifts in the weed flora of El-Menoufia Governorate where 67 species out of 146 species were recorded in our study while only 42 species out of 121 species were present in 1984 survey. Changes in the family composition, life form, life span, and chorology were reported. Reasons responsible for such changes have been discussed. Changes in the crop composition, cultivation area of each crop, excessive use of fertilizers and pesticides, changes in irrigation water quality and quantity are the leading change promoters. The high economic importance of the weed flora and the potential threats facing agriculture in Egypt necessitate the formulation of a sustainable strategy for maintaining agro-ecosystems of Egypt and its biodiversity.
\end{abstract}

Keywords: weed flora, arable lands, agricultural practices, Nile Delta.

\section{Introduction}

Agriculture in Egypt is as old as history and agricultural practices remained with no major changes for millennia (Bowman and Rogan, 1999). Modern agriculture in Egypt may be divided into two episodes separated by the establishment of the High Dam of Aswan (HDA), after which several transformations in agricultural practices took place (Biswas, 1993). Water availability allowed more areas to be cultivated and more crops to expand, especially those with high water needs such as rice and sugarcane (Abu-Zeid and El-Shibini, 1997). Even after liberalization of crop cultivation in 1990 this trend continued to increase (Barnes, 2014) (Table 1).

On the other side, around 130 million tons of mineral-rich sediments used to enrich the fertile arable lands of Egypt annually were trapped in Lake Nasser since 1969 (White, 1988). In order to compensate the degraded soil fertility, farmers started to rely heavily on chemical fertilizers which in return increased soil salinity and with the lack of proper drainage system, many areas were vulnerable to be salinized and ruined (Zaghloul, 2013). More areas were cultivated with salt tolerant crops such as rice but with its high water needs, salinity and water waste problem increased. Richards and Waterbury (1990) estimated that Egypt has spent more money on building drainage system than the money spent on building the HDA itself.

Population growth has also increased very rapidly from 30 million inhabitants in 1965 to around 98 million inhabitants in 2017 (World Bank, 2018). Egypt has the lowest arable land per capita of any country in Africa (Biswas, 1993). The population increase has triggered a strong wave of urban encroachment on the agricultural lands, especially after the 2011 revolution (Khamis et al., 2015 and Mohamed, 2017). These encroachments took place mainly in the most productive parts of the old lands of Egypt (Zaghloul, 2013). This has led to the reclamation of new lands in order to compensate the loss in the old lands, nevertheless of the fact that reclamation of new land is costing much more money and less effective than conserving the old productive one (Biswas, 1993 and Storkey et al., 2012). Lacking of proper infrastructure to contain these anthropogenic activities, especially in the heavily populated areas, has led to several environmental problems (Nagajyoti et al., 2010; Alloway, 2012; Wu et al., 2016 and Steffan et al., 2017). Consequences include water and soil contamination by sewage and industrial wastes and the accumulation of heavy metals in agricultural soil and subsequently in various plantations (Zhou et 


\section{Changes in Weed Flora in Response to Agricultural Practices in Egypt}

al., 2016; Wang et al., 2017; Chen et al., 2018 and Khalifa and Gad, 2018). These problems have also provided a better environment for pests and disease vectors to flourish and accordingly the use of pesticides has also intensified (Biswas, 1993 and El-Nemr, 2017). It is estimated that one million ton of pesticides has been injected into the Egyptian environment since the establishment of the HDA (Mansour, 2008). All of the previously mentioned changes have severely affected agriculture in Egypt (Hegazy et al., 2004; Brand et al., 2007 and El-Nemr, 2017).

In an agroecosystem, nothing could be a better indicator of changes than weeds. Weeds are the only component of the agroecosystems to resist human activities. Their persistence against elimination endeavors is truly remarkable (El-Demerdash et al., 1997). Survival techniques that weeds may use include high seed germination rate, short life cycle, efficient seed dispersal methods, allelopathy, and tolerance to different biotic and abiotic stresses (Baker, 1974). On the other hand, the heavy anthropogenic activities are severely affecting weed distribution and existence. Changes in the weed flora have been used to track the footprints of the past by several authors. Fahmy (1997) studied archeological weed flora of Egypt from Predynastic to GraecoRoman times (4500 B.C. - 395 A.D.) and related his findings to old agricultural practices. El-Hadidi (1993) studied the weed flora during the Neolithic / Predynastic period (4500-3100 B.C.) and concluded that the presence of some geographical elements today may be due to the former contact between different civilizations in Egypt's history. Recently, several studies have related the changes in the weed flora of arable lands to agricultural practices. Baessler and Klotz (2006) studied the effects of farming practices after World War II on the weed flora of Central Germany and for 50 years period. Andreasen and Streibig (2010) studied the changes in the weed flora of arable lands of Nordic countries in relation to changes after the World War II and the modern reformations in agricultural practices. El-Saied et al. (2015) studied the changes in the weed flora in Siwa Oasis in relation to farming practices. Richner et al. (2015) reported that the arable flora has dramatically altered in response to changing in agricultural practices since the Second World War. Ramôa et al. (2017) studied the weed flora changes in response to the modern reformations in agriculture in Portugal. Changes in agriculture practices don't only affect the weed flora of agroecosystems but extends to all biotic and abiotic components (Chamberlain et al., 2000).

Weed flora surveys in Egypt date back to the first half of the twentieth century (Simpson, 1932 and Andrews, 1945). Several studies have followed this early attempts by investigating the weed flora of some parts of the old lands of Egypt (Boulos, 1966 and 1967; El-Shayeb, 1989; Shaltout et al., 1992; Mashaly, 2003; Sadek, 2008 and El-Halawany et al., 2010) while others have investigated the weed flora of the reclaimed lands (Abd Elghani et al., 2013) and others have investigated the weed flora of the Egyptian Oases (Abd ElGhani, 1981, 1985 and 1998; Elsaied, 2012; El-Saied et al., 2015). Some studies have dealt with weeds associated with certain crops or plantations (Tadros and Atta, 1958; Imam and Kosinova, 1972; El-Kady et al., 1999; Turki and Sheded, 2002 and Mashaly and Awad, 2003) while others have dealt with seasonality of weeds (Zaki and Mashaly, 1992; El-Shayeb, 1984 and Ziada et al., 2007). Some studies gave a general view of the whole weed flora of the country (El-Hadidi and Kosinova, 1971; Boulos and El-Hadidi, 1984 Abd El-Ghani and El-Sawaf, 2004 and Shaltoot et al., 2010).

Several objectives and methodologies applied in various surveys made it hard to have a trustworthy comparison. One of the early studies of the weed flora in the Nile Delta is the study carried out by El-Shayeb (1984). ElShayeb (1984) studied the weed flora of ElMenoufia Governorate after 15 years of the establishment of the HDA where water availability and soil quality have changed agricultural practices, permitted more crop diversity, and consequently enriched the weed flora of the arable lands of Egypt. Agricultural practices remained very dynamic in response to the later deterioration in soil quality and water availability and quality. The present study aims at studying changes in the weed flora of El-Menoufia Governorate, south of the Nile Delta of Egypt after 34 years of El-Shayeb study in 1984 and around 50 years after the establishment of the HDA. Changes in the number of species, family composition, and geographical elements and life forms are to be detected and potential reasons for such changes if present, are to be investigated. 


\section{EISaied and Bedair}

Table 1. Cultivated areas of the major crops (feddans), total cultivated area of Egypt (feddans) and total population (millions) at three time points (1965, 1985, and 2016).

\begin{tabular}{|l|c|c|c|}
\hline & 1965 & 1985 & 2016 \\
\hline Rice & 641,000 & $1,042,000$ & $1,353,477$ \\
\hline Sugarcane & 111,000 & 248,000 & 325,912 \\
\hline Wheat & $1,500,000$ & $1,345,000$ & $3,353,151$ \\
\hline Maize & $1,850,000$ & $1,831,000$ & $1,741,000$ \\
\hline Clover & $2,362,000$ & $2,834,000$ & $1,562,000$ \\
\hline Cotton & $1,791,000$ & $1,296,000$ & 241,000 \\
\hline Total cultivated area & $5,500,000$ & $5,943,000$ & $8,961,000$ \\
\hline Total population & $30,875,964$ & $50,204,985$ & $95,688,681$ \\
\hline
\end{tabular}

\section{Study area}

According to (FAO, 2005) arable lands of Egypt may be divided into four groups: 1Old lands of the Nile Valley and Delta, 2Newly reclaimed lands around the Nile, 3Oases lands irrigated by the Nubian Sandstone Aquifer System (NSAS) and 4- Rain-fed lands along the Mediterranean coast of Egypt to the East and West of Nile Delta. El-Menoufia Governorate is located in the southern part of the Nile Delta of Egypt between long $30^{\circ} 15^{\prime}$ and $31^{\circ} 15^{\prime} \mathrm{E}$ and Lat. $30^{\circ} 10^{\prime}$ and $30^{\circ} 45^{\prime} \mathrm{N}$ with a total area of $2543 \mathrm{~km}^{2}$ and 3,941,293 inhabitants (El-Menoufia Governorate information Center, 2017). El-Menoufia is located between the two branches of the Nile Rosetta and Damietta. El-Menoufia Governorate bounded by Gharbiya Governorate to the north, Al Qalyubia Governorate to the east, Giza Governorate to the South and El Beheira Governorate to the west. El-Menoufia Governorate includes nine centers; Tala, El-Shohadaa, Menouf, Qwaisna, Berket AlSaba, Shebeen El-Koum, Al-Bagour, Ashmoun and El-Sadat. Main crops in ElMenoufia Governorate are wheat, clover, maize, and potato in addition to cotton, vegetables, and fruits. The agricultural land in El-Menoufia Governorate is of high quality (FAO, 2005). Common problems of agriculture in El-Menoufia are not isolated from Egypt's agricultural problems. Water shortage, soil degradation, blockage of drainage and irrigation networks, excessive use of plant hormones, chemical fertilizers, and pesticides.

\section{Materials and methods}

The present study of El-Menoufia Governorate was conducted between October 2016 and March 2018 to represent the flora of El-Menoufia agroecosystems. A total of 240 stands were selected to investigate the weed flora of the study area (120 stands in spring and another 120 in summer) (Fig. 1). Stands were chosen to represent different crops growing in the study area. Spring stands included 46 clover stands, 30 wheat stands, 26 potato and vegetables stands, and 18 orchards. Summer stands included 77 maize stands, 27 potato and vegetables, and 16 orchards. Stands were distributed along the nine centers according to the size of the cultivated area within each center. Each stand encompassed four quadrates $(5 \mathrm{~m} * 5 \mathrm{~m})$. GPS coordinates of each stand were recorded. Presence/absence data have been recorded for each species in each stand. All plant species existing in each stand were listed after complete identification according to Täckholm (1974) and Boulos (1999-2009). Voucher herbarium specimens were incorporated in the herbarium of the Department of Botany, Faculty of Science, AlAzhar University. Life form categories were identified after Raunkiaer (1934). Variation in the life form in the field was not considered. Phytogeographical affinity of each species was identified after the system of Eig (1931). Records of 1984 were acquired from ElShayeb (1984) and species list was prepared for further comparison with the present study findings. 


\section{Changes in Weed Flora in Response to Agricultural Practices in Egypt}

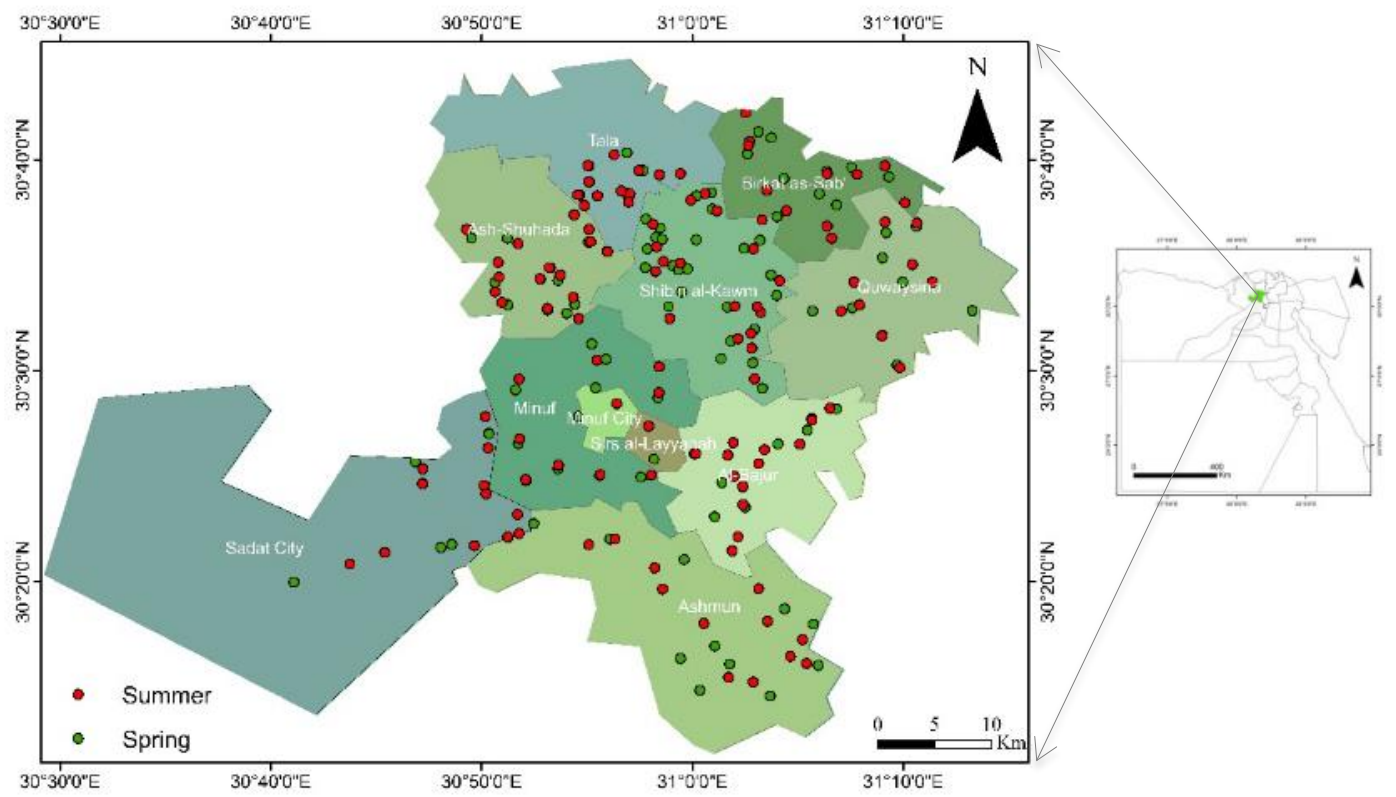

Fig. 1. Administrative map showing nine centers of El-Menoufia Governorate and the 240 selected stands in both spring and summer seasons.

\section{Results}

\section{Weed flora present status (2018)}

A total of 146 taxa belonging to 36 families and 105 genera were recorded in ElMenoufia Governorate in the present study (Table 1). Poaceae, Asteraceae, and Fabaceae were the most common families with 29 species (19.86\%), 19 species (13\%) and 11 species (7.53\%) of species composition, respectively. Brassicaceae, Amaranthaceae, Solanaceae, Chenopodiaceae, Euphorbiaceae and Convolvulaceae were represented by five to ten taxa each. Nine families were represented by two to four taxa while the rest 18 families were represented by only one species (Table 2 and Fig. 2).

With regard to life span, the majority of the recorded species in this study were annuals with 100 species (68.5\%), followed by the perennials with 44 species (30.1\%). Only two biennial species was recorded (Petroselinum crispum and Silybium marianum) (Table 2).

According to the classification of Raunkiaer (1934), eight life forms were recorded; therophytes were the most abundant life form and constituted $69.2 \%$ of total recorded species (101 species), followed by hemicryptophytes $(21$ species $=14.4 \%)$, chamaephytes ( 6 species $=4.1 \%$ ), geophytes $(5$ species $=3.4 \%)$, phanerophytes $(4$ species $=$ $2.7 \%)$, , four species from four different genera were helophytes.
(Lemna gibba, Eichhornia crassipes, Typha domingensis and Ranunculus rionii) and parasites were represented by two species (Cuscuta campestris and Orobanche cernua) (Tables 2 and Fig. 3).

From a phytogeographical point of view, cosmopolitan species represented 18.5\% of the recorded species with 27 species followed by paleotropical species with 25 species (17.1\%) and pantropical with 12 species (8.2\%) in addition to a single endemic (Sonchus macrocarpus) and neotropical (Amaranthus spinosus) species. The rest 80 species were classified into three groups; monoregional, biregional or pluriregional. A total of 19 species representing $13 \%$ of the total number of recorded species were monoregional of different affinities. Biregional geoelements were represented by 31 species forming $21.2 \%$ of recorded species. Pluriregional geoelements were represented by 30 species forming $20.5 \%$ of recorded species (Tables 2 and Fig. 4).

\section{Changes in the weed flora (1984 - 2018)}

Results of El-Shayeb (1984) were used to evaluate changes in weed flora during the period from 1984 to 2018. Number of species recorded in the present study was 146 while 121 species were recorded in 1984 . Number of shared species was 79 while 67 species were recorded only in the present study and 42 species were recorded only in 1984. 


\section{ElSaied and Bedair}

Table 2. List of species recorded in the agro-ecosystem of El-Menoufia Governorate. The species referred to their families, and life span; Annual=Ann, Biennial=Bie, Perennial=Per. according to Boulos (1999-2009), life forms according to (Raunkiaer, 1934); Therophyte=Th, Hemicryptophyte=Hem, Chamaephyte=Cha, Phanerophyte $=\mathrm{Ph}, \mathrm{Parasite}=\mathrm{Par}$, Geophyte=Geo, Helophyte=Hel, Hydrophytes= Hyd. Phytogeographical affinities according to the system of Eig (1931); COSM=Cosmopolitan, ER-SR=Euro-Siberian, IR-TR= Irano-Turanian, ME=Mediterranean, NEO=Neotropical, PAL=Paleotropical, PAN=Pantropical, SA-SI=Saharo-Sindian, S-Z=Sudano-Zambesian. Species were categorized into three groups; species recorded in the present study only (A), species recorded in 1984 only (B), Species recorded in both studies (A, B). Families are arranged alphabetically within each category; genera and species are in alphabetical order within their respective families.

\begin{tabular}{|c|c|c|c|c|c|}
\hline Species & Family & Life span & Life form & Floristic categories & Record \\
\hline Alternanthera sessilis (L.) DC. & Amaranthaceae & Per. & Hel. & PAN & A \\
\hline Amaranthus caudatus L. & Amaranthaceae & Ann. & Th. & COSM & A \\
\hline Amaranthus spinosus L. & Amaranthaceae & Ann. & Th. & NEO & $\mathrm{A}$ \\
\hline Amaranthus tricolor L. & Amaranthaceae & Ann. & Th. & PAL & A \\
\hline $\begin{array}{l}\text { Petroselinum crispum (Mill.) } \\
\text { Nyman ex A.W. Hill } \\
\end{array}$ & Apiaceae & Bie. & Th. & ME & A \\
\hline Cynanchum acutum L. & Asclepiadaceae & Per. & Hem. & ME + IR-TR + ER-SR & A \\
\hline Anthemis retusa Delile & Asteraceae & Ann. & Th. & ME + IR-TR + SA-SI & A \\
\hline Bidens pilosa L. & Asteraceae & Ann. & Th. & PAN & A \\
\hline Glebionis coronaria (L.) Tzvelev & Asteraceae & Ann. & Th. & ME & A \\
\hline $\begin{array}{l}\text { Launaea mucronata (Forssk.) } \\
\text { Muschl. }\end{array}$ & Asteraceae & Ann. & Th. & SA-SI & A \\
\hline Launaea nudicaulis (L.) Hook. f. & Asteraceae & Per. & Hem. & IR-TR & A \\
\hline Pluchea dioscoridis (L.) DC. & Asteraceae & Per. & $\mathrm{Ph}$. & SA-SI + S-Z & A \\
\hline $\begin{array}{l}\text { Pulicaria undulata (L.) } \\
\text { C.A.Mey. }\end{array}$ & Asteraceae & Per. & Cha. & SA-SI + S-Z & A \\
\hline Scolymus maculatus L. & Asteraceae & Ann. & Th. & $\mathrm{ME}$ & A \\
\hline Senecio aegyptius L. & Asteraceae & Ann. & Th. & SA-SI & A \\
\hline $\begin{array}{l}\text { Sonchus macrocarpus Boulos \& } \\
\text { C.Jeffrey }\end{array}$ & Asteraceae & Ann. & Th. & Endemic & A \\
\hline Camelina rumelica Velen. & Brassicaceae & Ann. & Th. & $\mathrm{ME}$ & $\mathrm{A}$ \\
\hline $\begin{array}{l}\text { Stellaria pallida (Dumort.) } \\
\text { Murb. }\end{array}$ & Caryophyllaceae & Ann. & Th. & $\mathrm{ME}+\mathrm{ER}-\mathrm{SR}$ & A \\
\hline Vaccaria pyramidata Medik. & Caryophyllaceae & Ann. & Th. & ME + IR-TR + ER-SR & $\bar{A}$ \\
\hline Atriplex lindleyi Moq. & Chenopodiaceae & Per. & Cha. & $\mathrm{ME}+\mathrm{SA}-\mathrm{SI}$ & $\bar{A}$ \\
\hline Chenopodium glaucum L. & Chenopodiaceae & Ann. & Th. & ME + ER-SR & $\mathrm{A}$ \\
\hline Cuscuta campestris Yunck. & Convolvulaceae & Ann. & Par. & PAN & $\mathrm{A}$ \\
\hline Ipomoea cairica (L.) Sweet & Convolvulaceae & Per. & Hem. & PAL & $\mathrm{A}$ \\
\hline Ipomoea eriocarpa R. Br. & Convolvulaceae & Ann. & Th. & PAL & A \\
\hline Cyperus alopecuroides Rottb. & Cyperaceae & Per. & Hel. & PAN & A \\
\hline Cyperus articulatus L. & Cyperaceae & Per. & Hel. & PAN & A \\
\hline Cyperus digitatus Roxb. & Cyperaceae & Per. & Geo. & ME + IR-TR + ER-SR & A \\
\hline Ricinus communis L. & Euphorbiaceae & Per. & Ph. & PAL & A \\
\hline Alhagi graecorum Boiss. & Fabaceae & Per. & Hem. & PAL & A \\
\hline Medicago sativa L. & Fabaceae & Per. & Hem. & ME + IR-TR + ER-SR & A \\
\hline Scorpiurus muricatus L. & Fabaceae & Ann. & Th. & ME & A \\
\hline Trigonella $s p$ & Fabaceae & Ann. & Th. & ME & A \\
\hline Mentha longifolia (L.) Huds. & Lamiaceae & Per. & Hem. & $\begin{array}{l}\text { PAL } \\
\end{array}$ & $\mathrm{A}$ \\
\hline Mentha pulegium L. & Lamiaceae & Per. & Hem. & ME + IR-TR + ER-SR & $\bar{A}$ \\
\hline Lemna gibba L. & Lemnaceae & Per. & Hyd & COSM & $\mathrm{A}$ \\
\hline Glinus lotoides L. & Molluginaceae & Ann. & Th. & ME + IR-TR & $\mathrm{A}$ \\
\hline Orobanche cernua Loefl. & Orobanchaceae & Per. & Par. & ME + IR-TR & $\mathrm{A}$ \\
\hline Bromus catharticus Vahl & Poaceae & Ann. & Th. & ME + IR-TR + ER-SR & A \\
\hline Bromus diandrus Roth. & Poaceae & Ann. & Th. & ME + IR-TR & $\mathrm{A}$ \\
\hline $\begin{array}{l}\text { Dactyloctenium aegyptium (L.) } \\
\text { Willd. }\end{array}$ & Poaceae & Ann. & Th. & PAL & A \\
\hline $\begin{array}{l}\text { Desmostachya bipinnata (L.) } \\
\text { Stapf }\end{array}$ & Poaceae & Per. & Hem. & SA-SI + S-Z & A \\
\hline
\end{tabular}




\section{Changes in Weed Flora in Response to Agricultural Practices in Egypt}

\begin{tabular}{|c|c|c|c|c|c|}
\hline $\begin{array}{l}\text { Echinochloa crusgalli (L.) P. } \\
\text { Beauv. }\end{array}$ & Poaceae & Ann. & Th. & PAL & A \\
\hline Hordeum marinum L. & Poaceae & Ann. & Th. & $\begin{array}{c}\mathrm{ME}+\mathrm{IR}-\mathrm{TR}+\mathrm{ER}-\mathrm{SR}+ \\
\mathrm{PAL}\end{array}$ & A \\
\hline $\begin{array}{l}\text { Imperata cylindrica (L.) } \\
\text { Raeusch. }\end{array}$ & Poaceae & Per. & Hem. & ME + IR-TR + SA-SI & A \\
\hline Leptochloa fusca (L.) Kunth & Poaceae & Per. & Hem. & PAL & A \\
\hline Lolium perenne L. & Poaceae & Per. & Hem. & COSM & $\mathrm{A}$ \\
\hline Panicum coloratum L. & Poaceae & Per. & Geo. & ME & $\mathrm{A}$ \\
\hline Paspalum distichum L. & Poaceae & Per. & Hem. & PAL & $\mathrm{A}$ \\
\hline $\begin{array}{l}\text { Pennisetum divisum (J.F.Gmel.) } \\
\text { Henrard }\end{array}$ & Poaceae & Per. & Hem. & SA-SI & A \\
\hline Phalaris paradoxa L. & Poaceae & Ann. & Th. & ME + IR-TR & $\mathrm{A}$ \\
\hline $\begin{array}{l}\text { Phragmites australis (Cav.) Trin. } \\
\text { ex Steud. }\end{array}$ & Poaceae & Per. & Geo. & PAL & A \\
\hline Sorghum bicolor (L.) Moench & Poaceae & Ann. & Th. & PAL & $\mathrm{A}$ \\
\hline Polygonum maritimum L. & Polygonaceae & Per. & Hem. & $\mathrm{ME}$ & $\mathrm{A}$ \\
\hline Polygonum plebeium R. Br. & Polygonaceae & Per. & Cha. & PAL & $\mathrm{A}$ \\
\hline $\begin{array}{l}\text { Eichhornia crassipes (C. Mart.) } \\
\text { Solms }\end{array}$ & Pontederiaceae & Per. & Hyd & PAL & A \\
\hline Ranunculus rionii Lagger & Ranunculaceae & Ann. & Th. & ME + ER-SR & A \\
\hline Rosa sp & Rosaceae & Per. & $\mathrm{Ph}$. & IR-TR & $\mathrm{A}$ \\
\hline Veronica persica Poir. & Scrophulariaceae & Ann. & Th. & COSM & A \\
\hline Hyoscyamus muticus L. & Solanaceae & Per. & Hem. & $\mathrm{ME}$ & $\mathrm{A}$ \\
\hline Physalis angulata $\mathrm{L}$. & Solanaceae & Ann. & Th. & PAN & $\mathrm{A}$ \\
\hline Solanum villosum (L.) Mill. & Solanaceae & Ann. & Th. & ME + IR-TR + ER-SR & A \\
\hline Withania obtusifolia Täeckh. & Solanaceae & Per. & Ph. & ME + IR-TR & A \\
\hline Withania somnifera (L.) Dunal & Solanaceae & Per. & Cha. & SA-SI + S-Z & A \\
\hline $\begin{array}{l}\text { Reaumuria hirtella Jaub. \& } \\
\text { Spach. }\end{array}$ & Tamaricaceae & Per. & Cha. & IR-TR & A \\
\hline $\begin{array}{l}\text { Tamarix nilotica (Ehrenb.) } \\
\text { Bunge }\end{array}$ & Tamaricaceae & Per. & Ph. & SA-SI + S-Z & A \\
\hline $\begin{array}{l}\text { Typha domingensis (Pers.) Poir. } \\
\text { ex Steud. }\end{array}$ & Typhaceae & Per. & Hel. & PAN & A \\
\hline Tribulus pentandrus Forssk. & Zygophyllaceae & Ann. & Th. & SA-SI & $\mathrm{A}$ \\
\hline Tribulus terrestris L. & Zygophyllaceae & Ann. & Th. & IR-TR + ER-SR + S-Z & $\mathrm{A}$ \\
\hline Trianthema portulacastrum L. & Aizoaceae & Ann. & Th. & PAN & $\mathrm{A}, \mathrm{B}$ \\
\hline Amaranthus hybridus L. & Amaranthaceae & Ann. & Th. & COSM & $\mathrm{A}, \mathrm{B}$ \\
\hline Amaranthus retroflexus L. & Amaranthaceae & Ann. & Th. & COSM & $\mathrm{A}, \mathrm{B}$ \\
\hline Amaranthus viridis L. & Amaranthaceae & Ann. & Th. & COSM & A, B \\
\hline Ammi majus L. & Apiaceae & Ann. & Th. & ME + IR-TR & $\mathrm{A}, \mathrm{B}$ \\
\hline Ammi visnaga (L.) Lam. & Apiaceae & Ann. & Th. & ME + IR-TR & $\mathrm{A}, \mathrm{B}$ \\
\hline Foeniculum vulgare Mill. & Apiaceae & Ann. & Th. & ME + IR-TR & $\mathrm{A}, \mathrm{B}$ \\
\hline Cichorium endivia L. & Asteraceae & Ann. & Th. & ME + IR-TR & $\mathrm{A}, \mathrm{B}$ \\
\hline $\begin{array}{l}\text { Conyza bonariensis (L.) } \\
\text { Cronquist }\end{array}$ & Asteraceae & Ann. & Th. & $\mathrm{ME}$ & A, B \\
\hline $\begin{array}{l}\text { Pseudognaohalium luteo-album } \\
\text { (L.) Hilliard \& B. L. Burtt. }\end{array}$ & Asteraceae & Ann. & Th. & $\mathrm{ME}+\mathrm{IR}-\mathrm{TR}+\mathrm{SA}-\mathrm{SI}$ & A, B \\
\hline Senecio glaucus L. & Asteraceae & Ann. & Th. & IR-TR + SA-SI & $\mathrm{A}, \mathrm{B}$ \\
\hline Senecio vulgaris L. & Asteraceae & Ann. & Th. & ME + IR-TR + ER-SR & $\mathrm{A}, \mathrm{B}$ \\
\hline Silybum marianum (L.) Gaertn. & Asteraceae & Bie. & Th. & ME + IR-TR + ER-SR & $\mathrm{A}, \mathrm{B}$ \\
\hline Sonchus oleraceus L. & Asteraceae & Ann. & Th. & COSM & $\mathrm{A}, \mathrm{B}$ \\
\hline $\begin{array}{l}\text { Urospermum picroides (L.) } \\
\text { Scop. ex F.W.Schmidt }\end{array}$ & Asteraceae & Ann. & Th. & $\mathrm{ME}+\mathrm{IR}-\mathrm{TR}$ & A, B \\
\hline Xanthium spinosum L. & Asteraceae & Ann. & Th. & PAL & $\mathrm{A}, \mathrm{B}$ \\
\hline Brassica nigra (L.) K.Koch & Brassicaceae & Ann. & Th. & ME + ER-SR & $\mathrm{A}, \mathrm{B}$ \\
\hline Brassica rapa L. & Brassicaceae & Ann. & Th. & ME + ER-SR & $\mathrm{A}, \mathrm{B}$ \\
\hline
\end{tabular}


ElSaied and Bedair

\begin{tabular}{|c|c|c|c|c|c|}
\hline $\begin{array}{l}\text { Capsella bursa-pastoris (L.) } \\
\text { Medik. }\end{array}$ & Brassicaceae & Ann. & Th. & COSM & A, B \\
\hline Coronopus didymus (L.) Sm. & Brassicaceae & Ann. & Th. & COSM & $\mathrm{A}, \mathrm{B}$ \\
\hline $\begin{array}{l}\text { Coronopus squamatus (Forssk.) } \\
\text { Asch. }\end{array}$ & Brassicaceae & Ann. & Th. & ME + IR-TR + ER-SR & A, B \\
\hline Eruca sativa Mill. & Brassicaceae & Ann. & Th. & $\begin{array}{c}\text { ME + IR-TR + ER-SR + } \\
\text { SA-SI } \\
\end{array}$ & A, B \\
\hline Raphanus raphanistrum L. & Brassicaceae & Ann. & Th. & ME + ER-SR & A, B \\
\hline Sisymbrium irio L. & Brassicaceae & Ann. & Th. & ME + IR-TR + ER-SR & A, B \\
\hline Silene rubella L. & Caryophyllaceae & Ann. & Th. & ME & $\mathrm{A}, \mathrm{B}$ \\
\hline Spergularia marina (L.) Griseb. & Caryophyllaceae & Ann. & Th. & COSM & $\mathrm{A}, \mathrm{B}$ \\
\hline Beta vulgaris L. & Chenopodiaceae & Ann. & Th. & ME + IR-TR + ER-SR & $\mathrm{A}, \mathrm{B}$ \\
\hline Chenopodium album L. & Chenopodiaceae & Ann. & Th. & COSM & $\mathrm{A}, \mathrm{B}$ \\
\hline Chenopodium ambrosioides L. & Chenopodiaceae & Ann. & Cha. & PAL & A, B \\
\hline Chenopodium murale L. & Chenopodiaceae & Ann. & Th. & COSM & $A, B$ \\
\hline Convolvulus arvensis L. & Convolvulaceae & Per. & Hem. & PAL & $\mathrm{A}, \mathrm{B}$ \\
\hline Ipomoea carnea Jacq. & Convolvulaceae & Per. & Ph. & ME + IR-TR & A, B \\
\hline Cyperus rotundus L. & Cyperaceae & Per. & Geo. & PAN & A, B \\
\hline Euphorbia helioscopia L. & Euphorbiaceae & Ann. & Th. & COSM & A, B \\
\hline Euphorbia heterophylla L. & Euphorbiaceae & Ann. & Th. & PAN & $\mathrm{A}, \mathrm{B}$ \\
\hline Euphorbia indica Lam. & Euphorbiaceae & Ann. & Th. & SA-SI & A, B \\
\hline Euphorbia peplus L. & Euphorbiaceae & Ann. & Th. & COSM & $\mathrm{A}, \mathrm{B}$ \\
\hline Euphorbia prostrata Aiton & Euphorbiaceae & Ann. & Th. & COSM & $\mathrm{A}, \mathrm{B}$ \\
\hline Lotus glaber Mill. & Fabaceae & Per. & Hem. & ME + IR-TR + ER-SR & $\mathrm{A}, \mathrm{B}$ \\
\hline Medicago intertexta (L.) Mill. & Fabaceae & Ann. & Th. & ME + ER-SR & $\mathrm{A}, \mathrm{B}$ \\
\hline Melilotus indicus (L.) All. & Fabaceae & Ann. & Th. & PAL & $\mathrm{A}, \mathrm{B}$ \\
\hline Melilotus messanensis (L.) All. & Fabaceae & Ann. & Th. & $\mathrm{ME}+\mathrm{IR}-\mathrm{TR}$ & A, B \\
\hline Trifolium resupinatum L. & Fabaceae & Ann. & Th. & ME + IR-TR + ER-SR & $A, B$ \\
\hline Vicia monantha Retz. & Fabaceae & Ann. & Th. & ME + IR-TR & $A, B$ \\
\hline Vicia sativa $\mathrm{L}$. & Fabaceae & Ann. & Th. & ME + IR-TR + ER-SR & $\mathrm{A}, \mathrm{B}$ \\
\hline Fumaria densiflora DC. & Fumariaceae & Ann. & Th. & ME + IR-TR + ER-SR & $\mathrm{A}, \mathrm{B}$ \\
\hline Lamium amplexicaule L. & Lamiaceae & Ann. & Th. & ME + IR-TR + ER-SR & $\mathrm{A}, \mathrm{B}$ \\
\hline Emex spinosa (L.) Campd. & Loranthaceae & Ann. & Th. & ME + SA-SI & $\mathrm{A}, \mathrm{B}$ \\
\hline Abutilon theophrasti Medik. & Malvaceae & Ann. & Th. & ME + IR-TR + ER-SR & A, B \\
\hline Hibiscus trionum L. & Malvaceae & Ann. & Th. & PAL & $\mathrm{A}, \mathrm{B}$ \\
\hline Malva parviflora L. & Malvaceae & Ann. & Th. & ME + IR-TR & $\mathrm{A}, \mathrm{B}$ \\
\hline Oxalis corniculata L. & Oxalidaceae & Ann. & Th. & COSM & $\mathrm{A}, \mathrm{B}$ \\
\hline Plantago major L. & Plantaginaceae & Per. & Hem. & COSM & $\mathrm{A}, \mathrm{B}$ \\
\hline Avena fatua $\mathrm{L}$. & Poaceae & Ann. & Th. & COSM & A, B \\
\hline Avena sativa $\mathrm{L}$. & Poaceae & Ann. & Th. & COSM & $A, B$ \\
\hline $\begin{array}{l}\text { Brachiaria eruciformis (Sm.) } \\
\text { Griseb. }\end{array}$ & Poaceae & Ann. & Th. & ME + IR-TR & A, B \\
\hline Cynodon dactylon (L.) Pers. & Poaceae & Per. & Geo. & PAN & $\mathrm{A}, \mathrm{B}$ \\
\hline Digitaria sanguinalis (L.) Scop. & Poaceae & Ann. & Th. & PAL & A, B \\
\hline Dinebra retroflexa (Vahl) Panz. & Poaceae & Ann. & Th. & PAL & A, B \\
\hline Echinochloa colona (L.) Link & Poaceae & Ann. & Th. & ME + IR-TR + PAL & $\mathrm{A}, \mathrm{B}$ \\
\hline Phalaris minor Retz. & Poaceae & Ann. & Th. & ME + IR-TR & $\mathrm{A}, \mathrm{B}$ \\
\hline Poa annua L. & Poaceae & Ann. & Th. & ME + IR-TR + ER-SR & $\mathrm{A}, \mathrm{B}$ \\
\hline $\begin{array}{l}\text { Polypogon monspeliensis (L.) } \\
\text { Desf. }\end{array}$ & Poaceae & Ann. & Th. & COSM & A, B \\
\hline $\begin{array}{l}\text { Polypogon viridis (Gouan) } \\
\text { Breistr. }\end{array}$ & Poaceae & Ann. & Th. & ME + IR-TR + ER-SR & A, B \\
\hline $\begin{array}{l}\text { Setaria verticillata (L.) P. } \\
\text { Beauv. }\end{array}$ & Poaceae & Ann. & Th. & COSM & A, B \\
\hline Setaria viridis (L.) P. Beauv. & Poaceae & Ann. & Th. & COSM & A, B \\
\hline Sorghum virgatum (Hack.) Stapf & Poaceae & Ann. & Th. & PAL & A, B \\
\hline $\begin{array}{l}\text { Persicaria salicifolia (Brouss. ex } \\
\text { Willd.) Assenov }\end{array}$ & Polygonaceae & Per. & Hem. & PAL & A, B \\
\hline
\end{tabular}




\section{Changes in Weed Flora in Response to Agricultural Practices in Egypt}

\begin{tabular}{|c|c|c|c|c|c|}
\hline Rumex dentatus L. & Polygonaceae & Ann. & Th. & ME + IR-TR + ER-SR & A, B \\
\hline Portulaca oleracea L. & Portulacaceae & Ann. & Th. & COSM & $\mathrm{A}, \mathrm{B}$ \\
\hline Anagallis arvensis L. & Primulacae & Ann. & Th. & COSM & A, B \\
\hline Veronica anagallis-aquatica L. & Scrophulariaceae & Per. & Hel. & COSM & $\mathrm{A}, \mathrm{B}$ \\
\hline Veronica polita Fr. & Scrophulariaceae & Ann. & Th. & ME + IR-TR + ER-SR & $A, B$ \\
\hline Datura stramonium L. & Solanaceae & Ann. & Th. & PAL & $A, B$ \\
\hline Solanum nigrum L. & Solanaceae & Ann. & Th. & COSM & $A, B$ \\
\hline Corchorus olitorius L. & Tiliaceae & Ann. & Th. & PAN & $\mathrm{A}, \mathrm{B}$ \\
\hline Urtica urens L. & Urticaceae & Ann. & Th. & ME + IR-TR + ER-SR & $\mathrm{A}, \mathrm{B}$ \\
\hline Phyla nodiflora (L.) Greene & Verbenaceae & Per. & Hem. & PAL & $\mathrm{A}, \mathrm{B}$ \\
\hline Amaranthus graecizans L. & Amaranthaceae & Ann. & Th. & COSM & $\mathrm{B}$ \\
\hline Torilis nodosa (L.) Gaertn. & Apiaceae & Ann. & Th. & ME + IR-TR + ER-SR & $\mathrm{B}$ \\
\hline $\begin{array}{l}\text { Centaurea calcitrapella Bornm. } \\
\text { \& Dinsm. }\end{array}$ & Asteraceae & Bie. & Cha. & ME + ER-SR & B \\
\hline Eclipta prostrata (L.) L. & Asteraceae & Ann. & Th. & PAL + NEO & $\mathrm{B}$ \\
\hline Ethulia conyzoides L.f. & Asteraceae & Ann. & Th. & $\overline{\text { PAL }}$ & $\mathrm{B}$ \\
\hline Sonchus asper (L.) Hill. & Asteraceae & Ann. & Th. & ME + IR-TR & $\mathrm{B}$ \\
\hline $\begin{array}{l}\text { Symphyotrichum squamatum } \\
\text { (Spreng.) G.L.Nesom }\end{array}$ & Asteraceae & Per. & Cha. & NEO & B \\
\hline Xanthium pungens Wallr. & Asteraceae & Ann. & Th. & S-Z & B \\
\hline Xanthium strumarium L. & Asteraceae & Ann. & Th. & $\mathrm{S}-\mathrm{Z}$ & $\mathrm{B}$ \\
\hline Brassica tournefortii Gouan & Brassicaceae & Ann. & Th. & ME + SA-SI & $\mathrm{B}$ \\
\hline Lepidium sativum L. & Brassicaceae & Ann. & Th. & COSM & $\mathrm{B}$ \\
\hline Sinapis alba L. & Brassicaceae & Ann. & Th. & ME + IR-TR + ER-SR & $\mathrm{B}$ \\
\hline Sinapis allionii Jacq. & Brassicaceae & Ann. & Th. & Endemic & $\mathrm{B}$ \\
\hline Sinapis arvensis L. & Brassicaceae & Ann. & Th. & IR-TR + ER-SR + ME & B \\
\hline Sinapis turgida (Pers.) Delile & Brassicaceae & Ann. & Th. & Endemic & $\mathrm{B}$ \\
\hline Stellaria media (L.) Vill. & Caryophyllaceae & Ann. & Th. & COSM & $\mathrm{B}$ \\
\hline Cuscuta pedicellata Ledeb. & Convolvulaceae & Ann. & Par. & IR-TR + ME & B \\
\hline Cuscuta planiflora Ten. & Convolvulaceae & Ann. & Par. & PAL & $\mathrm{B}$ \\
\hline $\begin{array}{l}\text { Chrozophora plicata (Vahl) } \\
\text { A.Juss. ex Spreng. }\end{array}$ & Euphorbiaceae & Ann. & Th. & $\mathrm{S}-\mathrm{Z}+\mathrm{PAL}$ & B \\
\hline Euphorbia terracina L. & Euphorbiaceae & Per. & Hem. & $\mathrm{ME}$ & $\mathrm{B}$ \\
\hline Lathyrus hirsutus L. & Fabaceae & Ann. & Th. & ME + IR-TR + ER-SR & $\mathrm{B}$ \\
\hline Lotus arabicus L. & Fabaceae & Ann. & Th. & S-Z + SA-SI & $\mathrm{B}$ \\
\hline Medicago polymorpha L. & Fabaceae & Ann. & Th. & ME + IR-TR + ER-SR & $\mathrm{B}$ \\
\hline Vicia cinerea M.Bieb. & Fabaceae & Ann. & Th. & ME + IR-TR & $\mathrm{B}$ \\
\hline Vicia lutea $\mathrm{L}$. & Fabaceae & Ann. & Th. & ME & $\bar{B}$ \\
\hline Vicia peregrina L. & Fabaceae & Ann. & Th. & ME + IR-TR & $\mathrm{B}$ \\
\hline Mentha microphylla K.Koch & Labiatae & Per. & Geo. & PAL & $\mathrm{B}$ \\
\hline Althaea ludwigii L. & Malvaceae & Ann. & Th. & SA-SI + IR-TR + S-Z & $\mathrm{B}$ \\
\hline Sida alba L. & Malvaceae & Ann. & Th. & PAN & $\mathrm{B}$ \\
\hline Orobanche crenata Forssk. & Orobanchaceae & Per. & Par. & ME + IR-TR & $\mathrm{B}$ \\
\hline Avena sterilis L. & Poaceae & Ann. & Th. & ME + IR-TR & $\mathrm{B}$ \\
\hline $\begin{array}{l}\text { Brachiaria reptans (L.) } \\
\text { C.A.Gardner \& C.E.Hubb. }\end{array}$ & Poaceae & Ann. & Th. & PAL & B \\
\hline $\begin{array}{l}\text { Dichanthium annulatum } \\
\text { (Forssk.) Stapf }\end{array}$ & Poaceae & Per. & Hem. & $\mathrm{PAL}+\mathrm{NEO}$ & B \\
\hline $\begin{array}{l}\text { Echinochloa stagnina (Retz.) } \\
\text { P.Beauv. }\end{array}$ & Poaceae & Per. & Hel. & PAL & B \\
\hline Leptochloa panicea (Retz.) Ohwi & Poaceae & Ann. & Th. & PAL & $\mathrm{B}$ \\
\hline Lolium multiflorum Lam. & Poaceae & Ann. & Th. & ME + ER-SR + IR-TR & B \\
\hline Lolium temulentum L. & Poaceae & Ann. & Th. & ER-SR + ME + IR-TR & $\mathrm{B}$ \\
\hline Panicum repens L. & Poaceae & Per. & Geo. & $\mathrm{PAL}+\mathrm{NEO}+\mathrm{ME}$ & $\mathrm{B}$ \\
\hline Phalaris canariensis L. & Poaceae & Ann. & Th. & $\mathrm{ME}$ & B \\
\hline Polygonum bellardii All. & Polygonaceae & Ann. & Th. & IR-TR + ME + ER-SR & $\mathrm{B}$ \\
\hline Veronica anagalloides Guss. & Scrophulariaceae & Per. & Geo. & ER-SR + ME + IR-TR & B \\
\hline Verbena supina L. & Verbenaceae & Ann. & Th. & $\mathrm{ME}+\mathrm{IR}-\mathrm{TR}+\mathrm{SA}-\mathrm{SI}$ & $\mathrm{B}$ \\
\hline
\end{tabular}




\section{ElSaied and Bedair}

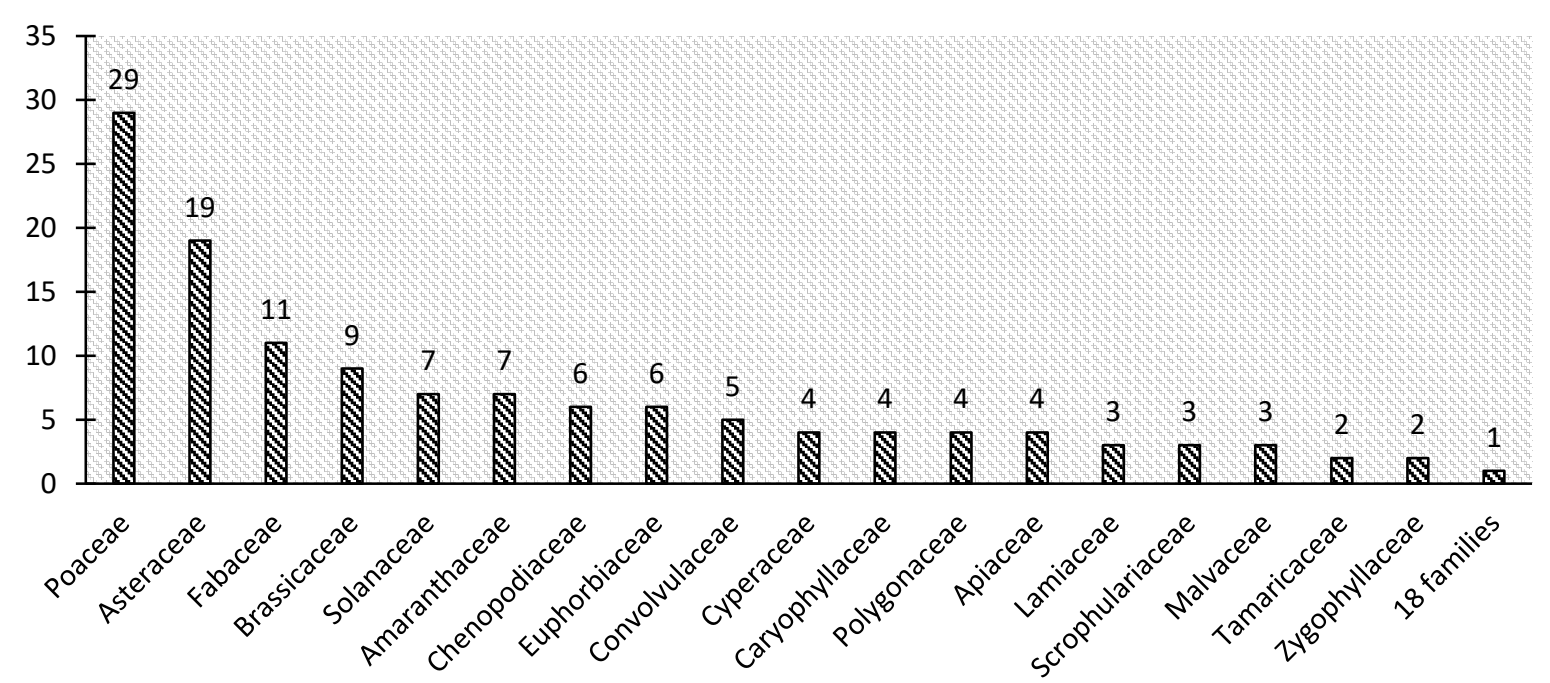

Fig. 2. Number of species recorded in each family collected from El-Menoufia Governorate in the present study.

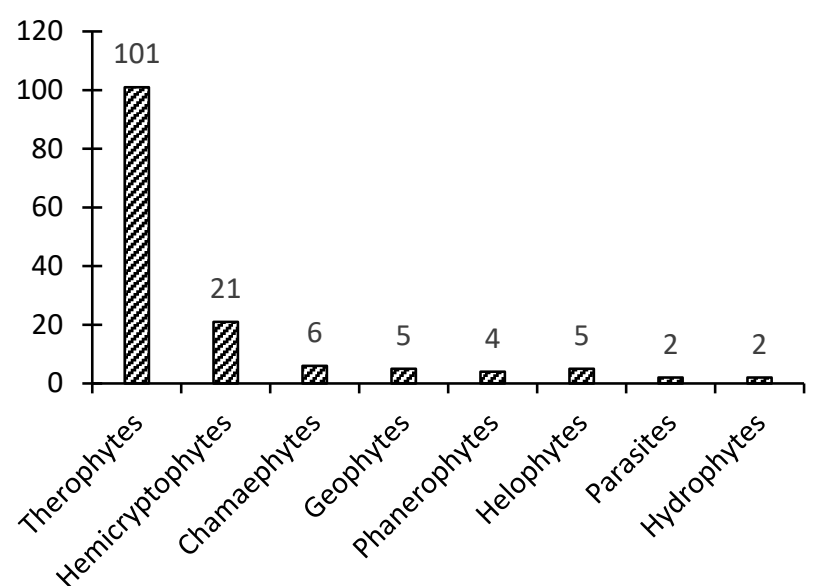

Fig. 3. Life forms of the species recorded in ElMenoufia Governorate in the present study

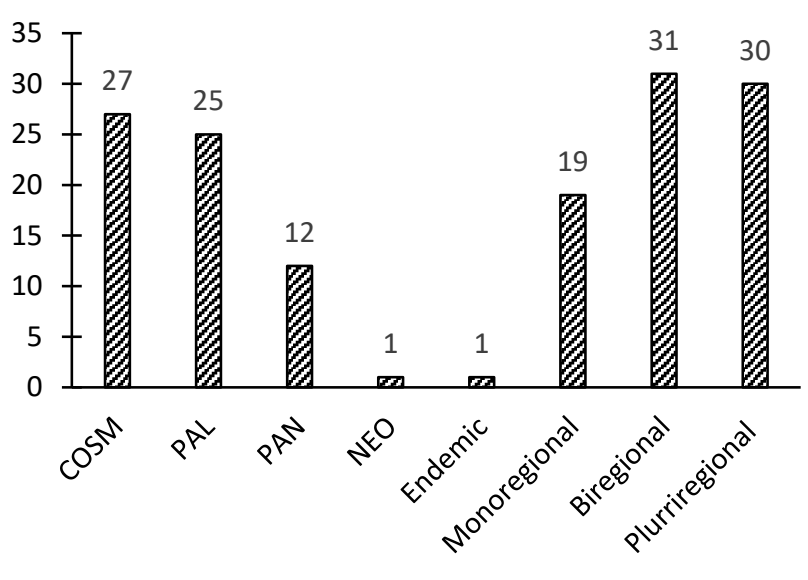

Fig. 4. Floristic categories of the species recorded in El-Menoufia Governorate in the present study

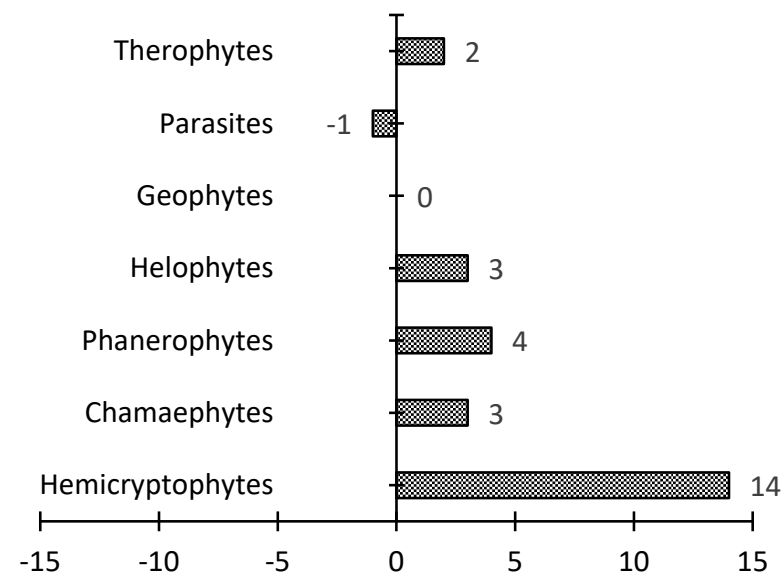

Fig. 5. Changes in the number of species belonging to different life forms during the period 1984- 2018

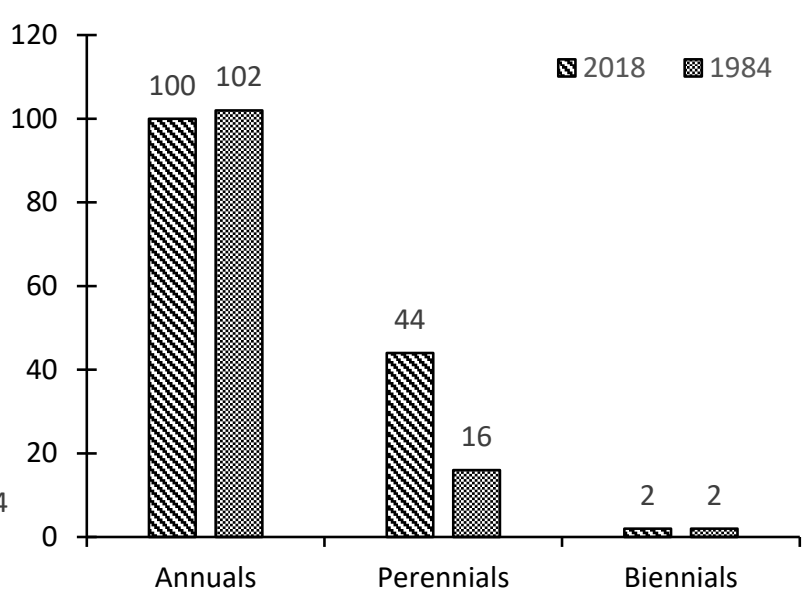

Fig. 6. Changes in the number of species belonging to different life spans during the period 1984- 2018 


\section{Changes in Weed Flora in Response to Agricultural Practices in Egypt}

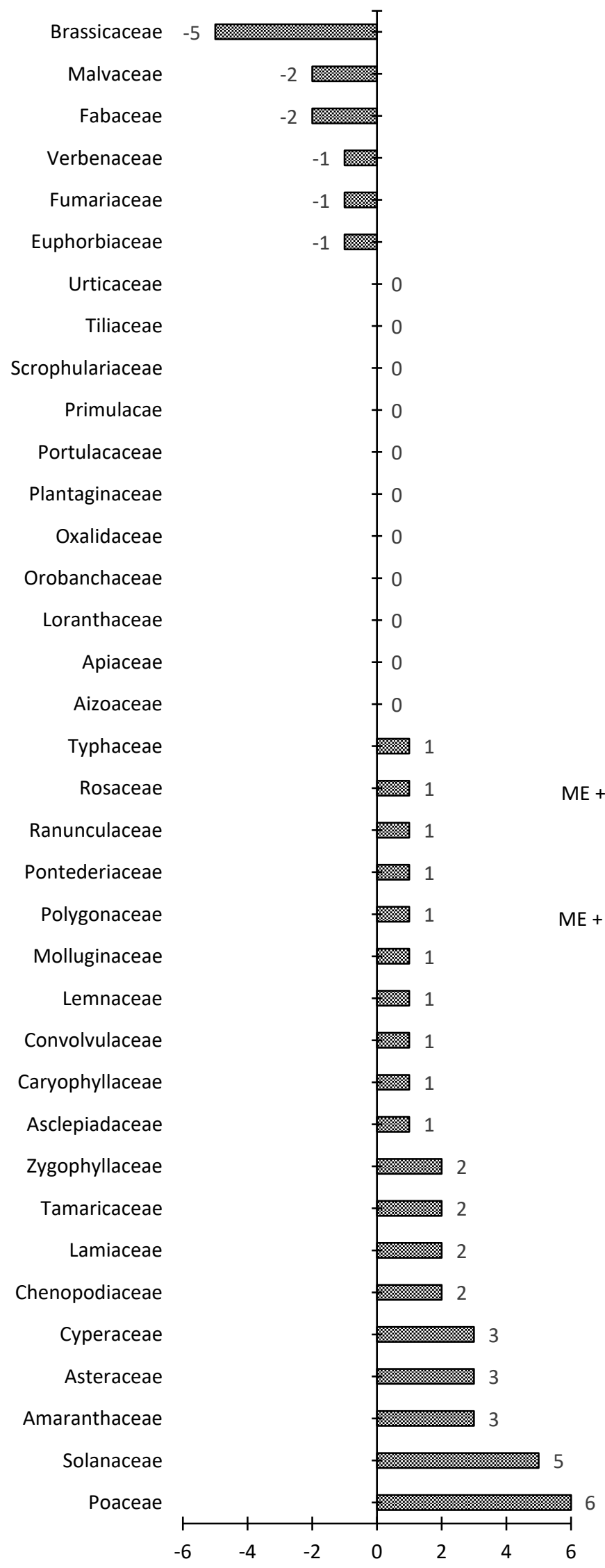

Fig. 7. Changes in the number of species belonging to different families during the period 1984- 2018

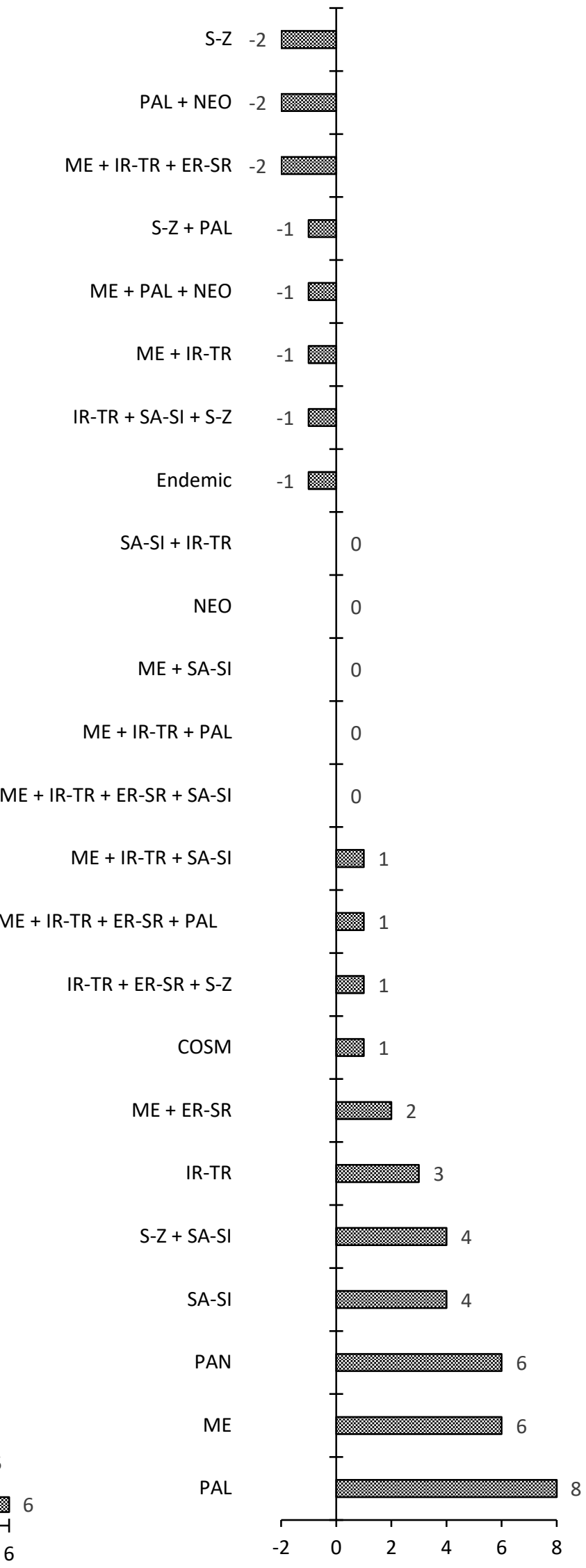

Fig. 8. Changes in the number of species belonging to different floristic categories during the period 1984- 2018 


\section{ElSaied and Bedair}

A total of 27 families were recorded in 1984 while 36 families were recorded in 2018. Nine families have been recorded only in this study (Tamaricaceae, Zygophyllaceae, Asclepiadaceae, Lemnaceae, Molluginaceae, Pontederiaceae, Ranunculaceae, Rosaceae, and Typhaceae). Poaceae, Asteraceae, Fabaceae and Brassicaceae were the most common families in both studies. However, number of species belonged to different families has changed where Brassicacea, Fabaceae and Malvaceae showed significant decrease while Poaceae, Solanaceae, Astraceae, Amaranthaceae and Cyperaceae showed a significant increase (Fig. 7 and Table 2).

Number of perennial species increased from 16 (1984) to 44 (2018) while number of annuals and biennials remained constant (Fig. 5). Hemicryptophytes have significantly increased from 7 in 1984 to 21 species in 2018 with $200 \%$ increase, while chamaephytes have doubled the number of species recorded in 1984 (Fig. 6).

Phytogeographical affinities showed various changes among different categories. Generally, Pantropical, Paleotropical, SaharoSindian and Mediterranean elements have increased significantly. On the level of monoregional elements, species belonging to Sudano-Zambesian region were recorded in El-Shayeb study only while Irano-Turanean elements were only recorded in the present study. On the level of the biregional and plurriregional elements, no major changes have been detected except for the Saharo Sindian elements that increased from 8 to 13 species (Fig. 8).

\section{Discussion}

The present study aimed at evaluating the weed flora changes in El-Menoufia Governorate, south of the Nile Delta of Egypt in response to different agricultural practices during the period from 1984 to 2018. ElShayeb (1984) studied the weed flora of ElMenoufia Governorate after 15 years of the establishment of the HDA where new agricultural practices have been adopted while some of the old practices were still in action. After five decades of establishing the HDA and after three decades of El-Shayeb's study, the present study seemed to be a logical point in time to evaluate changes that took place in the agro-ecosystems of Egypt especially in the most fertile part of the country.
Results showed that significant changes took place in the weed flora during this period on the level of family composition, number of species, life forms, life spans, and chorology. Several reasons may explain these alterations:

1- Total cultivated area in 1984 was 320,000 feddan with $88 \%$ of El-Menoufia Governorate $\left(1512 \mathrm{~km}^{2}\right)$ while today the total cultivated area has increased to 391,037 feddan with around $65 \%$ of El-Menoufia Governorate $\left(2543 \mathrm{~km}^{2}\right)$ after the inclusion of El-Sadat center in 1992 with a total area of around 1000 $\mathrm{km}^{2}$. The xero-halophyte species, Reaumuria hirtella, was only recorded in the fields of ElSadat, which is quite understandable as it is the desert back of El-Menoufia Governorate and the only place that may provide similar conditions to Reaumuria hirtella natural habitat. On the other side, urban encroachment on the expense of arable lands has replaced one fifth of the old fertile lands of El-Menoufia Governorate (Abd El-Fatah, 2013) which may contribute to the disappearance of the 42 species recorded in 1984 only.

2- Major crops cultivated in El-Menoufia in 1984 were wheat, clover, potato, soybean, broad bean and flax in winter and rice, cotton, soybean and maize in summer. In 2018 major crops are wheat, clover, and potato in winter while maize and potato are the major crops in summer. Broad bean, soybean, flax, cotton, and rice are cultivated in very limited scattered areas. El-Shayeb (1984) recorded Sinapis sp. as a unique associate species to flax. Sinapis sp. was not recorded in the present study and flax cultivation has declined in El-Menoufia Governorate to less than 100 feddans in 2018 and in the whole country from 60 thousand feddans in 1980s to 7 thousand feddans in 2016 (El-Nagdy et al., 2010 and CAPMAS, 2017). Cotton was reported as the cash crop of ElMenoufia Governorate in 1984 but governmental control of prices and problems related to production have forced farmers to replace cotton by crops like clover where they could get the best benefit of it. Accordingly the area of cotton cultivation has declined from 1.3 million feddans in 1985 to 241 thousand feddans in 2016 (Zaghloul, 2013 and CAPMAS, 2017). On the other hand, rice cultivation was banned in El-Menoufia Governorate in 2013 because of water scarcity. The ban was expanded in 2018 to restrict rice cultivation in the whole country from 1.4 million feddans in 2016 to 700 thousand feddans because of the potential water 


\section{Changes in Weed Flora in Response to Agricultural Practices in Egypt}

problems related to the establishment of the Ethiopian Renaissance Dam (CAPMAS, 2017).

3- Water scarcity have forced some farmers to use both drainage water and sub-surface water in the irrigation process. After liberalization of crops in 1990s, farmers choose to cultivate water intensive profitable crops like rice that led to the increase of water demands and forced farmers to use drainage water (Barnes, 2014).

4- After the blockage of Nile sediments, the use of chemical fertilizers has increased. According to FAO, the use of fertilizers in Egypt has increased from 750 ton/year in the 1990s to 2000 ton /year in the last ten years. Excessive use of chemical fertilizers has serious impacts on agroecosystems including reducing number of micro-fauna as well as increasing the concentrations of potassiun, phosphorus, calcium and magnesium in the top soils while reducing nitrate, calcium and magnesium in the sub-soils (Edmeades, 2003) and subsequently affecting the weed flora. Several studies have reported the bad effects on soil, crops, animals and human health (WQU, 2004; Taha et. al., 2012 and El-Bady, 2014).

5- Excessive use of herbicides to control weeds has a major role in determining weed flora composition, diversity and abundance (Sher and Al-Yemeny, 2011). Andreasen et al., (1996) stated that the intensive use of herbicides resulted in massive shifts in the weed flora of Europe. On the other side, the use of herbicides has developed herbicide-resistant weeds (Binimelis et al., 2009 and Heap 2010). Results showed significant increase in the number of hemicryptophytes and Poaceae members which may refer to the environmental deterioration in the Egyptian agroecosystems (Londe and Silva, 2014). Hemicryptophytes are considered the most resistant life form (Liddle and Greig-Smith, 1975; Hall and Kuss, 1989 and Cole, 1995) while Poaceae is a well-known family for its tolerance against drought, salinity, freeze and different abiotic stresses (Céccoli et. al., 2015; Fabbri et. al., 2016 and Landi et. al., 2017).

6- The establishment of the HDA has affected the flow of seeds from Africa towards Egypt. Results showed the absence of SudanoZambesian elements recorded by El-Shayeb in 1984. Fahmy (1997) stated that the establishment of dams along the Nile River has affected the distribution of weeds like Ceruana pratensis and Potentilla supine that disappeared from the silty banks of the river.
On the other side, results showed the increase of the Mediterranean and Irano-Turanean elements. Willcox (2012) reported the increase of weed migration among Europe, Middle East and Mediterranean region in the last decades because of the increase of transcontinental commerce and migration.

7- Other reasons may include the methodology and prospective of each study. El-Shayeb (1984) has investigated 40 stands with five stands for each center of El-Menoufia on a monthly basis for a whole year in order to investigate the reproductive capacity of ElMenoufia Governorate weed flora. In our study, 240 stands were investigated during spring and summer seasons with 120 stands for each season distributed along the nine centers of El-Menoufia Governorate in order to document the present situation of the weed flora. This may explain part of the large number of the newly recorded species (67) but does not explain the absence of 42 species, which may be due to the previously mentioned reasons.

Hassib (1951) recorded 395 species in Nile Delta while Shaltout et al. (2010) recorded 534 species. The present study added 18 new species to the flora of the Nile Delta (Amaranthus caudatus, Amaranthus tricolor, Petroselinum crispum, Anthemis retusa, Launaea mucronata, Pulicaria undulata, Scolymus maculatus, Camelina rumelica, Atriplex lindleyi, Scorpiurus muricatus, Mentha pulegium, Polygonum maritimum, Rosa sp., Veronica persica, Solanum villosum, Withania obtusifolia, Reaumuria hirtella and Tribulus pentandrus) and 67 species to ElMenoufia. On the other side, one third of the weed flora recorded by El-Shayeb in 1984 was not recorded in the present study in spite of the large number of investigated stands (Table 2). Different agricultural practices discussed above may have led to these results. Several studies have reported similar results from different areas around the world (Andreasen $e t$ al., 1996; Heap, 2010; Richner et al., 2015 and Ramôa et al., 2017).

\section{Conclusion and Recommendations}

Dramatic changes in the weed flora of El-Menoufia Governorate during the last thirty-four years necessitate the sustainable management of the arable lands of Egypt. Several problems are facing agriculture and without controlling such problems, serious impacts on the weed flora are to be expected. Saving this rich arable flora is not a luxury and 


\section{ElSaied and Bedair}

should be a priority as a part of our role in conserving biodiversity and within the frame of the Egyptian Government commitment to Rio de Janeiro Convention on Biological Diversity (CBD) and the UN 2030 Agenda for sustainable development.

It is very important to note that in spite of the fact that the heavy use of herbicides and other chemicals since 1984 may have eliminated number of sensitive weed species but the total number of weeds in the study area has increased. The current agricultural practices are neither helping farmers protecting their crops from weeds nor helping the survival of the weed flora. It became clear that the more destructive methods farmers use against weeds, the more weed resistance they get back. Several countries have developed various balanced strategies that took into consideration farmers' interests and conservation of biodiversity at the same time. Examples include compensation of farmers, prohibiting chemical spraying of field margins to give some space for the weed flora of the arable lands and strictly managing the use of different pesticides to avoid weed resistance development and all related environmental problems (Kleijn and Sutherland, 2003, Kleijn et al., 2011 and Richner et al., 2015).

A new chapter in the history of agriculture in Egypt is about to begin after the establishment of Ethiopian Renaissance Dam which threaten Egypt's share of Nile water (Zenawi, 2011a \& b). Egyptian Government is already taking steps forward to minimize water waste and consumption in agriculture but it is crucial to have a sustainable strategy to manage the entire agro-ecosystem biotic and abiotic components.

\section{Acknowledgements}

Authors would like to express their deep appreciation for the assistance of Prof. Dr. Adel Ibrahim Hamed El-Gazzar for his generous help with the plant identification and confirmation of the collected species. Our deep appreciation for the help of Prof. Dr. Fathy ElShayeb for allowing access to 1984 results and thoughtful discussions about changes in the weed flora in El-Menoufia Governorate. Special thanks to prof. Dr. Om Mohamed Ahmed Khafagi and Dr. Abdou Marie Hamed for thoughtful discussions and critical reading of the manuscript.

\section{References}

Abd El-Fatah, A. (2013). Urban Sprawl on Cultivated Land in El-Menofiya Governorate a Geographical Study, Using Geographical Information Systems and Remote Sensing. Ms. Thesis, Faculty of Arts, Cairo University, Cairo, Egypt, 310 p.

Abd El-Ghani, M. (1981). Preliminary Studies on the Vegetation of Bahariya OasisEgypt. M.Sc. Thesis, Faculty of Science, Cairo University, Egypt, 618 p.

Abd El-Ghani, M. (1985). Comparative Study on the Vegetation of the Bahariya and Farafra Oases and the Faiyum Region. Ph.D Thesis, Faculty of Science, Cairo University, Cairo, Egypt, 464 p.

Abd El-Ghani, M. (1998). Environmental correlates of species distribution in arid desert ecosystems of eastern Egypt. J. Arid Environ, 38(2), 297-313.

Abd El-Ghani, M. and El-Sawaf, N. (2004). Diversity and distribution of plant species in agro-ecosystems of Egypt. Syst. Geogr. Plants. , 74 (2), 319-336.

Abd El-Ghani, M.; Soliman, A.; Hamdy, R. and Bennoba, E. (2013). Weed flora in the reclaimed lands along the northern sector of the Nile Valley in Egypt. Turk J Bot., 37(3), 464-488.

Abu-Zeid, M. and El-Shibini, F. (1997). Egypt's high Aswan dam. INT J WATER RESOUR D, 13(2), 209-218.

Alloway, B. (2012). Heavy Metals in Soils. Springer, Netherlands. 615 p.

Andreasen, C. and Streibig, J. C. (2011). Evaluation of changes in weed flora in arable fields of Nordic countries-based on Danish long-term surveys. Weed Res., 51(3), 214-226. Andreasen, C.; Stryhn, H. and Streibig. J. (1996). Decline of the flora in Danish arable fields. J. Appl. Ecol., 33: 619-626.

Andrews, F. (1945). Water plants in the Gezira canals: A study of aquatic plants and their control in the canals of the Gezira cotton area (Anglo-Egyptian Sudan). Ann Appl Biol., 32 (1), 1-14.

Baessler, C. and Klotz, S. (2006). Effects of changes in agricultural land-use on landscape structure and arable weed vegetation over the last 50 vears. Agric Ecosyst Environ., 115(14), 43-50.

Baker, H. (1974). The evolution of weeds. Annu. Rev. Ecol. Evol. Svst. 5. 1-24.

Barnes, J. (2014). Mixing waters: The reuse of agricultural drainage water in Egypt. Geoforum, 57, 181-191.

Binimelis, R.; Pengue, W. and Monterroso, I. (2009). "Transgenic treadmill”: responses to the emergence and spread of glyphosate- 


\section{Changes in Weed Flora in Response to Agricultural Practices in Egypt}

resistant johnsongrass in Argentina. Geoforum, 40(4), 623-633.

Biswas, A. (1993). Land Resources for Sustainable Agricultural Development in Egypt, Ambio 22(8), pp. 556-560.

Boulos, L. (1966). Flora of the Nile region in the Egyptian Nubia. Feddes Repert. 73, 184215.

Boulos, L. (1967). On the weed flora of Aswan, Egypt. Bot. not. 120, 368-372.

Boulos, L. (1999). Flora of Egypt. vol. I (Azollaceae- Oxalidaceae). A1-Hadara Publishing, Cairo, Egypt, 419 p.

Boulos, L. (2000). Flora of Egypt. vol. II (Geraniaceae-Boraginaceae). A1-Hadara Publishing, Cairo, Egypt, 352 p.

Boulos, L. (2002). Flora of Egypt. vol. III (Verbenaceae- Compositae). A1-Hadara Publishing, Cairo, Egypt, 373 p.

Boulos, L. (2005). Flora of Egypt. vol. IV Monocotyledons (AlismataceaeOrchidaceae). A1-Hadara Publishing, Cairo, Egypt, 617 p.

Boulos, L. (2009). Flora of Egypt Checklist (Revised Annotated ed.). Al-Hadara Publishing, Cairo, Egypt, 410 p.

Boulos, L. and El-Hadidi, M. (1984). The Weed Flora of Egypt. Cairo: The American University Cairo Press.178 p.

Bowman, A., and Rogan, E. (1999). Agriculture in Egvpt: from pharaonic to modern times. Proceedings of the British Academy, 96, 1-31.

Brand, J.; Yaduraju, N.; Shivakumar, B. and McMurray, L. (2007). Weed management. Lentil: An ancient crop for modern times.-Springer, Dordrecht, the Netherlands. pp 159-172.

CAPMAS (2017). Central Agency for Public Mobilization and Statistics. Statistical Year Book, Arab Republic of Egypt. Cairo.

Céccoli, G.: Ramos, J.: Pilatti, V.: Dellaferrera, I.; Tivano, J.; Taleisnik, E. and Vegetti, A. (2015). Salt glands in the Poaceae family and their relationship to salinity tolerance. Bot Rev, 81 (2), 162-178.

Chamberlain, D.; Fuller, R.; Bunce, R.; Duckworth, J. and Shrubb, M. (2000). Changes in the abundance of farmland birds in relation to the timing of agricultural intensification in England and Wales. J. Appl. Ecol., 37(5), 771-788.

Chen, L.; Zhou, S.; Wu, S.; Wang, C.; Li, B.; Li, Y. and Wang, J. (2018). Combining emission inventory and isotope ratio analyses for quantitative source apportionment of heavy metals in agricultural soil. Chemosphere, 204, 140-147.

Cole, D. (1995). Experimental trampling of vegetation. II. Predictors of resistance and resilience. J. Appl. Ecol., 215-224.

Edmeades, D. (2003). The long-term effects of manures and fertilisers on soil productivity and quality: a review. NUTR CYCL AGROECOSYS., 66 (2), 165-180.

Eig, A. (1931). Les elements et les groups phytogeogra-phique auxiliaries dans la flore palestiniene. Feddes Repert, 63, 470-496.

El Hadidi, M. (1993). A historical flora of Egypt, a preliminary survey. Biological anthropology and the study of Ancient Egypt, 144-155.

El-Bady, M. (2014). Spatial distribution of some important heavy metals in the soils south of Manzala Lake in Bahr El-Baqar region, Egypt. NJEAS, 2 (3), 1-15

El-Demerdash, M.; Hosni, H. and Al-Ashri, N. (1997). Distribution of the weed communities in the North East Nile Delta, Egypt. Feddes Repert, 108, 219-232.

El-Hadidi, M. and Kosinová, J. (1971). Studies on the weed flora of cultivated land in Egypt: preliminary survey. Mitteilungen der Botanischen Staatssammlung München. 10, 354-367.

El-Halawany, E.; Mashalv, I.; Abu-Ziada, M. and Abd El-Aal, M. (2010). Habitat and plant life in El-Dakahlyia Governorate, Egypt. JOESE, 39(1), 83-108.

El-Kady, H.; Shaltout, K. and Mousa, M. (1999). Diversity of weed communities in the common orchards of the Nile Delta. Journal of Union of Arab Bioloaists 9 (B). 149-168

El-Menoufia Governorate information Center (2017). Statement Provided by Support Centre and Decision-Making. El-Menoufia Governorate, Egypt, 5 p.

El-Nagdy, G. A., Nassar, D. M., El-Kady, E. A. and El-Yamanee, G. S. (2010). Response of flax plant (Linum usitatissimum L.) to treatments with mineral and bio-fertilizers from nitrogen and phosphorus. $J$ Am Sci, 6(10), 207-217.

El-Nemr, M. (2017). Applicability of Sustainable Agriculture in Egypt. In Negm, A. M., Omran, E. S. E., Awaad, H., and Abuhashim, M. (2018). Update, Conclusions, and Recommendations for Sustainability of the Agricultural Environment in Egypt: The SoilWater-Food Nexus.

El-Saied, A. (2012). Vegetation and Environmental Changes in Two Oases, Western Desert, Egypt. Ph.D. Thesis, Faculty of Science, Al- Azhar University, Cairo, Egypt, 308 p.

El-Saied, A.; El-Ghamery, A.; Khafagi, O.; Powell, O. and Bedair, R. (2015). Floristic diversity and vegetation analysis of Siwa Oasis: An ancient agro-ecosystem in Egypt's Western Desert. AOAS. 60 (2), 361-372. 


\section{ElSaied and Bedair}

El-Shayeb, F. (1984). Studies on Reproductive Capacity of Major Weeds in the Cultivated Land in El-Menoufia Governorate. M. Sc. Thesis, Menoufia University, Shebin El-Kom. pp. 397.

El-Shayeb, F. (1989). Studies in the weed flora of the Nile Delta. Ph. D. Thesis, Menoufia University, Shebin El-Kom. pp. 429 Fabbri, L.: Ploschuk, E.: López, M.: Insausti, P. and Rua, G. (2016). Freeze tolerance differs between two ecotypes of Paspalum vaainatum (Poaceae). Acta Bot Brasilica, 30 (1), 152-156.

Fahmy, A. (1997). Evaluation of the weed flora of Egypt from Predynastic to GraecoRoman times. Veg Hist Archaeobot. 6 (4), 241247.

FAO (2005). Fertilizer Use by Crop in Egypt. Land and Plant Nutrition Management Service Land and Water Development Division, Rome, $62 \mathrm{p}$.

Hall. C. and Kuss. F. (1989). Vegetation alteration along trails in Shenandoah National Park. Virginia. Biol Cons. 48, 211-227.

Hassib, M. (1951). Distribution of plant communities in Egypt. Fouad I University Press, 29, 59-261.

Heap, I. (2010). The International Survey of Herbicide Resistant Weeds. Online Available on http://www.weedscience.com.

Hegazy, A.; Fahmy, G.; Ali, M. and Gomaa, N. (2004). Vegetation diversity in natural and agro-ecosystems of arid lands. COMMUNITY ECOL, 5(2), 163-176.

Imam, M. and Kosinova, J. (1972). Studies on the weed flora of cultivated land in Egypt 2. Weeds of rice fields. Botanische Jahrbücher fur Systematik, Pflanzengeschichte und Pflanzengeographie. 92, 90- 107.

Khalifa, $M$ and Gad, A. (2018). Assessment of heavy metals contamination in agricultural soil of Southwestern Nile Delta, Egypt. SOIL SEDIMENT CONTAM, 27 (7), 619-642.

Khamis, R.; Ali, A. and Hahn, M. (2015). Assessing the Urban Encroachment Phenomenon in Egvpt Using Satellite Imagery. IJSER, 6(11), 1148-1159.

Kleijn, D. and Sutherland, W. (2003). How effective are European agri-environment schemes in conserving and promoting biodiversity?. J. Appl. Ecol., 40 (6), 947-969.

Kleijn, D.; Rundlöf, M.; Scheper, J.; Smith, H. and Tscharntke, T. (2011). Does conservation on farmland contribute to halting the biodiversity decline?. Trends Ecol Evolut, 26(9), 474-481.

Landi, S.; Hausman, J.; Guerriero, G. and Esposito, S. (2017). Poaceae vs. abiotic stress: focus on drought and salt stress, recent insights and perspectives. Front. Plant Sci, 8, 12-14.
Liddle, M. and Greig-Smith, P. (1975). A survey of tracks and paths in a sand dune ecosystem. II. Vegetation. J. Appl. Ecol., 12. 909-930.

Londe, V. and Silva, J. (2014). Characterization of Poaceae (grass) species as indicators of the level of degradation in a stretch of riparian forest in Matutina, Brazil. Act Bot Brasilica, 28(1), 102-108.

Mansour, S. (2008). Environmental impact of pesticides in Egvpt. In Rev Environ Contam Toxicol. 196, 1-51.

Mashaly, I. (2003). Phytosociological study on the weed flora of croplands in Kafr ElSheikh Governorate, Egypt. El Minia Science Bulletin Botany Section, 14 (2). 127-153.

Mashaly, I. and Awad, E. (2003). Weed flora of orchards in the Nile delta. Egvpt: floristic features. Asian J. Plant Sci., 2 (3), 314-324.

Mohamed, N. (2017). Land degradation in the Nile Delta. In The Nile Delta (pp. 235-264). Springer, Cham.

Nagajyoti, P.; Lee, K. and Sreekanth, T. (2010). Heavy metals, occurrence and toxicity for plants: a review. ENVIRON CHEM LETT, 8 (3), 199-216.

Ramôa, S.; Oliveira e Silva, P.; Travlos, I.; Vasconcelos, T.: Forte, P.. and Portugal, J. (2017). Evaluation of weed flora changes in Portugal in a 10 year basis. AJCS, 11(3), 322328.

Raunkiaer, C. (1934). The Plant Life Forms and Statistical Plant Geography. Oxford, Clarendon Press, 632 p.

Richards, A. (1982). Egypt's Agricultural Development, 1800-1980: Technical and Social Change. Boulder, CO: Westview Press. Richards, A. and Waterbury, J. (1990). A political economy of the Middle East. Finanças e Desenvolvimento

Richner, N.; Holderegger, R.; Linder, H.; and Walter, $T$. (2015). Reviewing change in the arable flora of Europe: a meta-analysis. WEED RES, 55(1), 1-13.

Sadek, M. (2008). Ecological Study on the Weed Flora in El Dakahlia Governorate, Egypt (Doctoral dissertation, Mansoura University, Egypt).

Shaltout, K.; Sharaf El-Din, A. and Ahmed, D. (2010). Plant Life in the Nile Delta. 244 p. Shaltout, K.; Sharaf El-Din, A. and ElFahar, R. (1992). Weed communities of the common crops in the Nile Delta region. Flora, 187, 329-339.

Sher, H., and Al-Yemenv, M. (2011). Ecological investigation of the weed flora in arable and non-arable lands of Al-kharj Area, Saudi Arabia. AJAR, 6(4), 901-906.

Simpson, N. (1932). A report on the weed flora of the irrigation channels in Egypt. 


\section{Changes in Weed Flora in Response to Agricultural Practices in Egypt}

Ministry of Public Works, Government Press, Cairo, pp. 124.

SIS (2018). State Information Service. Egypt in figures 2018. Agriculture $65-78$.

Steffan, J.; Brevik, E.; Burgess, L. and Cerda, A. (2017). The effect of soil on human health: an overview. Eur J Soil Sci, 69, 159171.

Storkey, J.; Meyer, S.; Still, K. and Leuschner, C. (2012). The impact of agricultural intensification and land-use change on the European arable flora. Proceedings of the Royal Society of London B, Biological Sciences, 279 (1732), 1421-1429.

Täckholm, V. (1974). Students' Flora of Egypt. Cairo University, printed by Cooperative Printing Company Beirut, 888 p.

Tadros, T. and Atta, A. (1958). The plant communities of barley fields and uncultivated desert areas of Mareotis (Egypt). Plant Ecol., 8 (3), 161-175.

Taha, A.; El-Shehawy, M.; Mosa, A. and EL-Komy, M. (2012). Suitability of drainage water for irrigation and its impact on wheat and clover crops at Northern Delta, Egyptian JSSAE, 3 (6), 655-668.

Turki, Z. and Sheded, M. (2002). Some observations on the weed flora of rice fields in the Nile Delta, Egypt. Fedes Repert, 113 (5-6), 394-403.

Wang, G.; Zhang, S.; Xiao, L.; Zhong, Q.; Li, L.; Xu, G.; Deng, O. and Pu, Y. (2017). Heavy metals in soils from a typical industrial area in Sichuan, China: spatial distribution, source identification, and ecological risk assessment. ESPR, 24 (20), 16618-16630.

White, G. (1988). The environmental effects of the high dam at Aswan. Environment, Science and Policy for Sustainable Development, 30 (7), 4-40.

Willcox, G. (2012). Searching for the origins of arable weeds in the Near East. Veg Hist Archaeobot. 21, 163-167.

World Bank (2018). World Population Prospects: $2017 \quad$ Revision (https://data.worldbank.org/country/egyptarab-rep).

WQU, (2004). Reuse of Drainage Water: Alternative Solutions for Stopped Reuse
Stations. Water Quality Unit (WQU), Ministry of Water Resources and Irrigation, Cairo.

Wu, J.; Song, J.; Li, W. and Zheng, M. (2016). The accumulation of heavy metals in agricultural land and the associated potential ecological risks in Shenzhen, China. ESPR, 23, 1428-1440.

Zaghloul, S. (2013). Consideration of the agricultural problems as a base of water resource management in Egypt. In Seventeenth International Water Technology Conference, IWTC17, Istanbul (pp. 5-7).

Zaki, M. and Mashaly, I. (1992). Seasonal aspects of the flora of the field crops, Nile Delta, Egypt. Bull. Fac. Sci., Mansoura University 19 (1), 188 - 202.

Zenawi, M. (2011a). Prime Minister Meles Zenawi gave contradictory answers for Egyptian National Television on October 10, 2011 where he underpinned the existence of cordial relations with both states but accused Egypt for conspiring against Ethiopia's national interests in an interview on April 2, 2011 for domestic Television and Radio journalists while he consistently assured relations of friendship with Sudan. However, his official policy statements at a state visit on September 16, 2011 in Cairo underscored general friendship and cooperation with either Egypt or Sudan.

Zenawi, M. (2011b). The Prime Minister of the Federal Government of Ethiopia informed the Ethiopian people in a televised speech detailing size and budget estimate of the Dam on April 4/2011 after he officially inaugurated the beginning of its construction.

Zhou, J.; Feng, K.; Pei, Z. and Lu, M. (2016). Pollution assessment and spatial variation of soil heavy metals in Lixia River Region of Eastern China. J Soils Sediments. 16 (3). 748-755.

Ziada, M.; Sherbenv, G.; Askar, M.; Adams, R.; Albavrak, S.; Aksov, A. and Alvarez-Castellanos, P. (2007). Seasonal aspects of the flora of the field crops, Nile Delta, Egypt. Asian J. Plant Sci., 13 (4-8), 804 p. 